\title{
Applications of Artificial Neural Networks: A Review
}

\author{
Rajan Dharwal* and Loveneet Kaur \\ Department of CSE, Lovely Professional University, Phagwara, Jalandhar - 144411, Punjab, India; \\ rajandharwal@gmail.com, loveneet.19341@lpu.co.in
}

\begin{abstract}
Objectives: The objective of this research paper is study the different methods related to artificial neural network used for prediction and detection of the lung cancer in its early stages so that survival rate of lung cancer patients can be increased. Methods: Lung cancer is the leading cause of death in India so early detection of lung cancer is very important. The detection and prediction of lung cancer was determined with image prepossessing method where segmentation, smoothing and enhancement steps were processed and features were extracted from images and stages of lung cancer were identified with suitable artificial neural network model and also survival rate of lung cancer patients was determined. Findings: Artificial neural network has a significant role in medical area. In these days most of the disease cure methods are process with the help of artificial intelligence to increase the performance of output. In lung cancer disease the artificial neural network model is very useful because detection of lung cancer in its early stages can be determine and it is very important to cure this disease initially because with the increasing stages of lung cancer it is very difficult to cure this disease and also the survival rate of lung cancer patients in higher stages is very low Improvements: The ultimate goal of this paper is to study different methods of Artificial Neural Networks model that can help for detection, prediction and find the survival rate of lung cancer patients.
\end{abstract}

Keywords: Artificial Intelligence, Artificial Neural Network, Application of Neural Networks

\section{Introduction}

Artificial intelligence is a part of computer science that leads to creation of intelligent system, which can work and reacts like humans. Artificial neural network is a non-linear computational system inspired by the structure, behaviour and learning abilities of a biological brain.

\subsection{Artificial Neural Networks}

The term "neural network" resulted from the research in artificial intelligence, which attempts to understand and model human brain functionality. Artificial intelligence is a part of computer science which focuses to creation of intelligent machines, that is, systems that work and behave like humans. From recent few years, artificial neural networks have proved themselves as a better alternative for solving complex problems in many areas. Input layer, hidden layers and output layer are the lay- ers of Artificial Neural Network. Hidden layers perform intermediate computation to produce required output from the various inputs received 2 . For pattern recognition applications, efficiency of neural network depends on the learning algorithm adopted. In supervised learning the correct answer is provided for every input to the network and Un-supervised learning in which result is derived from prior assumptions and inferences; however the correct result is not known to system and hybrid learning, which contains both supervised and unsupervised learning.

\section{Biological Neural Networks}

Human brain contains a billion of cells which are interconnected to receive process and transmit information. Each cell works as a simple processing unit and are core

${ }^{*}$ Author for correspondence 
components of nervous system. Interaction between these simple processing units, the brain manages to perform extremely difficult tasks.

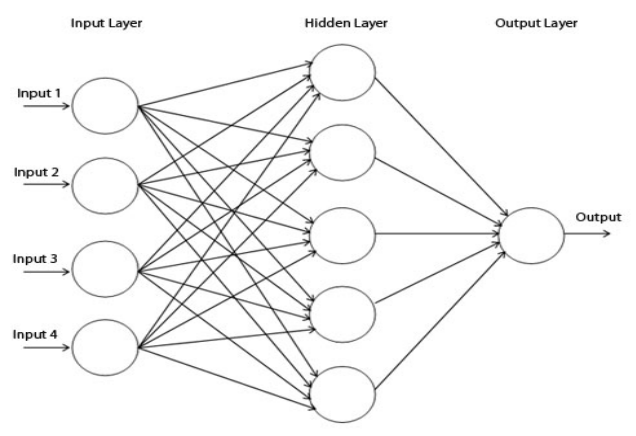

Figure 1. Neural network.

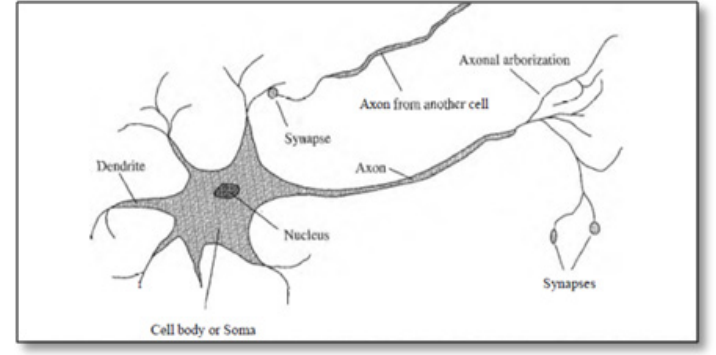

Figure 2. A biological neuron.

Biological neural networks are made up of these real biological neurons, one neuron makes connections with other neurons and signals are propagated between them. These signals control the activity of brain in central nervous system. Artificial neural networks are composed of interconnected artificial neurons, modelled on the interconnecting biological neurons in the human nervous system. Artificial neural networks can be used to understand the behaviour of human brain, or to solve complex problems.

\subsection{Elements of Artificial Neural Networks}

The fundamental building block of artificial neural network is neuron. Its components are valid whether neuron is used for input, output or in one of the hidden layers. A simple neural network consists of three layers, activation function, learning technique and weights. All these layers consist of neurons which are interconnected to form a network.

- Input Layer: Input layer is feature vector of problem. The extracted features are passing to input layer of neural network. It has the value in numeric form. Feature vector should be chosen very carefully. It should be able to describe the information properly and it should not contain any redundant information or faulty data, otherwise it will decrease the overall performance of the network. In short, input layer receives information and passes for further processing.

- Hidden Layers: A Hidden layer receives information from input layer and perform all the processes it. Hidden layers may vary from network to network that we selected. Number of hidden layers depends upon the nature and size of problem. For every problem, number of hidden layer have to be experimented and the set on the value for which the results are optimum.

- Output Layer: Output layer receives processed information from hidden layers and give results to the external user. Here an output layer depends on the outcome of the problem. For example if the problem is of classification then the output layer will give class number.

- Activation Function: Activation function exhibits a great variety and has a strong effect over the behaviour of neural network. The sum of weighted inputs is applied with an activation function to obtain the output response. The activation function is an abstraction representing the rate of firing in the cell. Most of the neural networks pass the output of layers through activation functions. Activation functions scale the output of neural network into proper range. For neurons present in the same layer, same activation function is used.

Most commonly used activation functions are:

- Linear Activation Function:

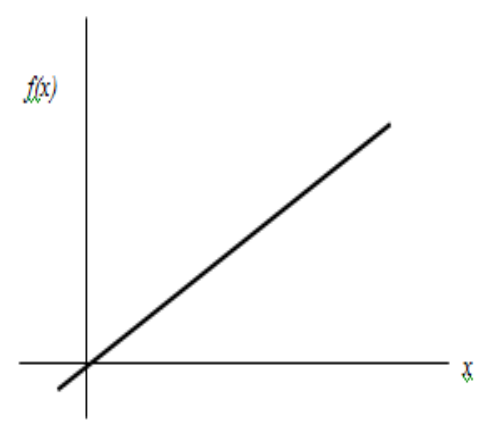

Figure 3. Identity activation function. 
These functions are most commonly used in single layer networks.

The expression for identity function is given by Equation

$\mathrm{F}(\mathrm{x})=\mathrm{x}$ for all $\mathrm{x}$

The output is continuously varying value as shown in Figure.

- Binary Step Activation Function:

The mathematical representation of binary step activation function is given by Equation.

$$
\begin{gathered}
\mathrm{F}(\mathrm{x})=1 \text { if } \mathrm{x} \geq \theta \\
=0 \text { if } \mathrm{x}<\theta
\end{gathered}
$$

Where, $\theta$ indicates the threshold value.

Figure shows its variation of output of function with input values.

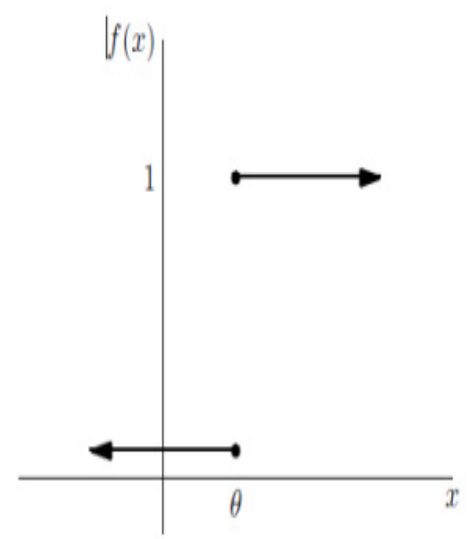

Figure 4. Binary step activation function.

- Log Sigmoid Transfer Function:

- This function yields output between the range 0 and 1 . The output is calculated according to Equation.

$$
\text { Logs }(\mathrm{n})=\frac{1}{1+\exp (-n)}
$$

- Hyperbolic Tangent Sigmoid Activation Function:

- This transfer function yields result scaled between -1 and 1 . The hyperbolic tangent function can be used with gradient descent based training methods as it has a derivative. The output is calculated according to Equation.

$$
\mathrm{n}=\frac{\mathbf{2}}{(1+\exp (-2 * n))}-\mathbf{1}
$$

\subsection{Learning in Artificial Neural Networks}

A learning system changes itself in order to adapt to various changes, e.g. environmental change. A processing unit is can change its input/output behaviour in accordance with the changes in environment. Learning method also required so that during training phase, weights can be modified in response to input/output changes. In artificial neural networks, learning can be supervised, unsupervised and reinforced.

- Supervised Learning: Supervised learning is also known as learning with teacher as an external teacher controls learning and incorporate global information. In supervised learning, the neural network is trained by providing both, the input vectors and the expected outputs. The difference between actual output and desired output serves as error measure. Figure 5 shows the block diagram of supervised learning.

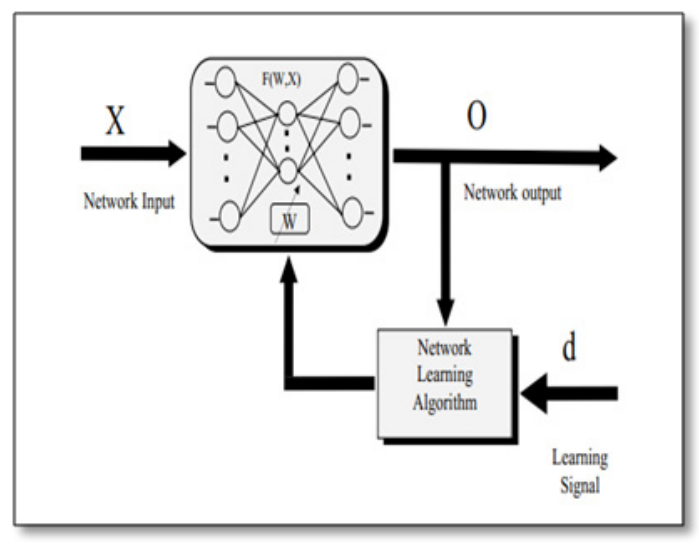

Figure 5. Supervised learning.

At each instant of time, input $X$ is applied to network and desired response $d$ of the system is provided by the external teacher. The distance between actual network output $O$ and desired response $d$ serves as error measure. The main objective of supervised learning is to reduce the difference between actual output and desired output. This is achieved by adjusting connection weights so that the network is more likely to produce output close to the desired value. The weights are adjusted continuously until the performance is satisfactory. Supervised learning is majorly used in prediction and classification problems.

- Unsupervised Learning: In this type of learning, only inputs are given and no desired output of the neural network. It is also called as learning 
without a teacher because there is no external teacher. Here learning, the network must discover any regularity, separating properties, etc. and network change its parameters accordingly to produce optimal results. Unsupervised learning is often used to perform clustering, i.e. unsupervised classification of input without any prior knowledge about actual classes. Figure 6 shows the block diagram of unsupervised learning.

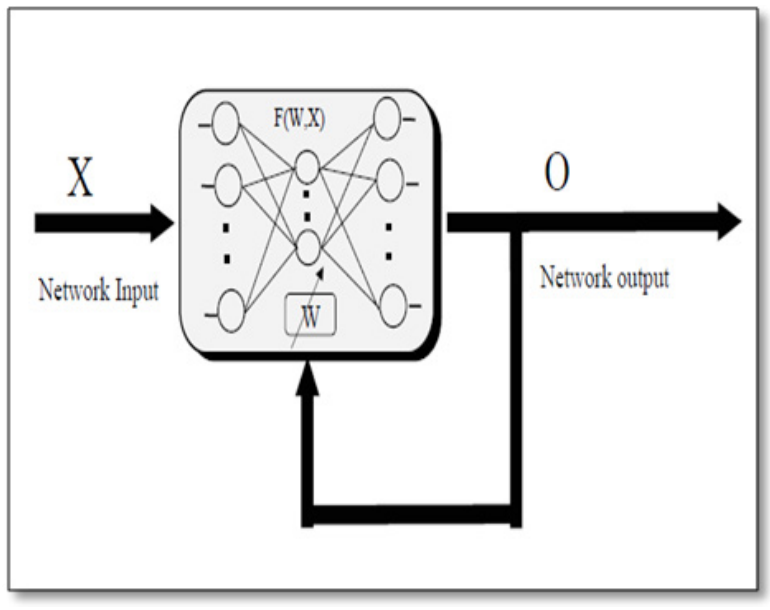

Figure 6. Unsupervised learning.

- Reinforcement Learning: In many situations, unlike unsupervised learning less detailed information is available and reinforcement learning is method is used to deal with such situations. In Reinforcement learning is a form of supervised learning where the network gets some feedback from environment. The feedback signal is in the form of only evaluative (yes/no) not instructive, i.e. the feedback signals only specify whether output is right or not. If the feedback signal says that output is wrong then it does not give any hint as what the right output should be.

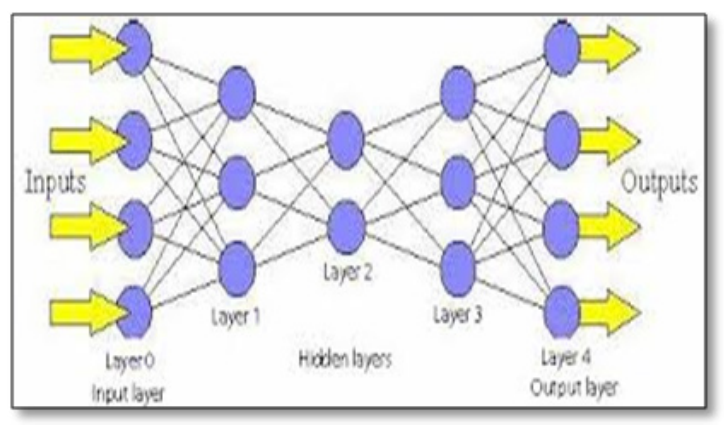

Figure 7. Feedback/Recurrent neural network.

\section{Neural Network Architecture}

There exist various neural network architectures. They differ from each other in many factors such as number of hidden layers, learning techniques, activation functions, etc. The two mostly used neural network architectures are:

\subsection{Feed forward Neural Networks}

These networks allow only one direction signal flow, i.e. from input nodes, through hidden nodes to output nodes. There is no feedback or loops in feed forward neural networks. In these networks, like any other neural network perceptron are organized into layers. The input and hidden layer must be connected and hidden layer can be connected to another hidden layer or output layer. The information is "feed forward" from one layer to another hence, called feed forward neural network.

\subsection{Feedback Neural Networks}

Feedback neural networks allow signals travelling in both directions by introducing loops in network.

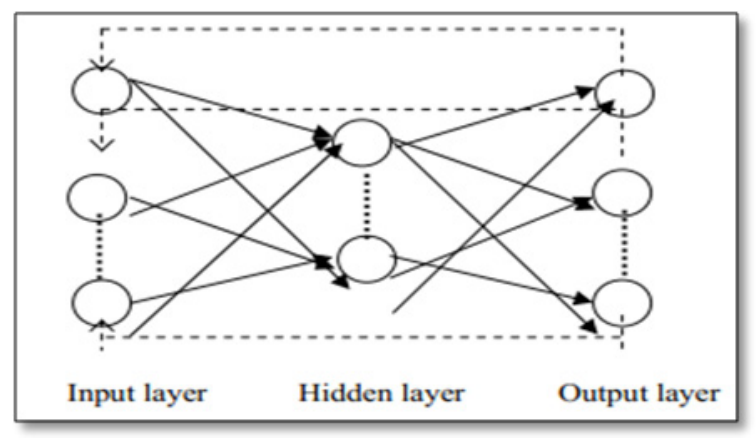

Figure 8. Recurrent/Feedback neural network.

There are feedback signals travelling from one layer to another. Feedback or recurrent networks are dynamic in nature, i.e. they change their state continuously until they get satisfactory response.

\subsection{Feed Forward Back Propagation Neural Network}

This network is composed of two neural network algorithms. The term "feed forward" refers the method from where the networks will be recognize a pattern and the term "back propagation" describes a process from where the networks will be train. In other words, "feed forward" 
describes how neural network processes and recalls patterns.

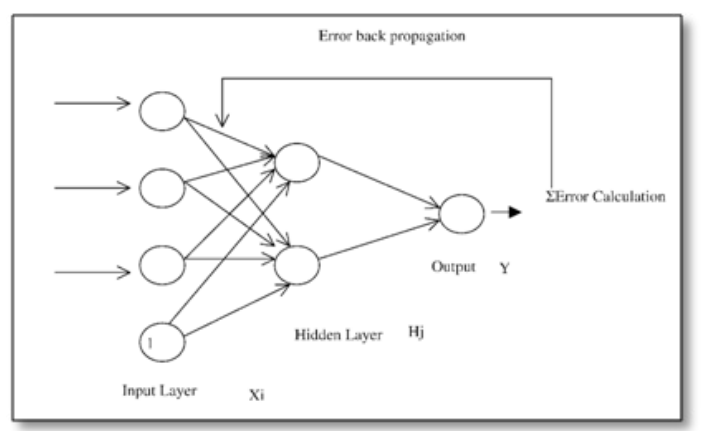

Figure 9. Feed forward back propagation neural network.

Back propagation is a form of supervised training, i.e. network must be provided with input as well as desired output. The desired outputs are compared with actual outputs to compute errors. Back propagation is a method which takes calculated error and then weights and input threshold of neural network are altered in a way that causes the error to be reduced.

\section{Use of Artificial Neural Networks in Lung Cancer Detection}

Lung cancer is a disease of uncontrolled cell growth in tissues of cells ${ }^{1}$. Lung cancer basically is of two types: Non-small lung cancer and Small cell lung cancer. Lungs are larger in size so tumour can grow inside for a long time even before they find through symptoms such as coughing and fatigue occurs. This disease is the leading cause of the deaths in worldwide. It is world wide spread disease that causes $13 \%$ deaths in 2008 and the rate is increasing rapidly every year. Therefore, the identification of this disease becomes very important. The Major cause of lung cancer is lack of awareness about lung cancer. Specially, in India the death rate of lung cancer is very high. In India it is the second largest disease which causes 0.3 million deaths in every year. Detection of lung cancer in the early stage is the key of cure of lung cancer disease and it is very challenging problem because the structure of cells that are overlapped with each other so, it is not easy to detect this disease. Although, in this disease survival rate of the patient decreases as age of patients increases.

In studied about gastric cancer is due to malignant growth of stomach that can develop anywhere inside the stomach 2 . It is at fourth position if we talk about the most common type of cancer found in worldwide. Author developed Artificial Neural Network system by analysed the data of 436 patients with stomach cancer from private organisation to find survival rate of gastric lung cancer. He found that the survival rate of gastric cancer by using COX propagation hazard. The author concluded that the survival rate of gastric cancer patients between years 2002 to 2007 was respectively $77.9 \%, 53.1 \%, 40.8 \%, 32 \%$ and $17.4 \%$.

Author evaluated the diagnosis potential of Artificial Neural Network model that was combined with six tumour markers in auxiliary diagnoses of lung cancer ${ }^{3}$. The system was trained with 242 samples including 93 lung cancer patients, 66 lung benign disease patients and 83 healthy people from a private institute. 19 parameters were used there like smoking, dust exposure, cooking fuel, chemical exposure etc. to differentiate the lung cancer from lung benign cancer and normal controls and sensitivity, specificity and accuracy was found $98.3 \%$, 99.5\% and $96.9 \%$ respectively. Another model with six tumours markers used to distinguish between lung cancer from gastric cancer by using three Artificial Neural Networks and sensitivity, specificity and accuracy was found $100 \%$, $83.5 \%$ and $93.5 \%$ respectively.

Author used back propagation algorithms in two phases feed forward and back propagation procedure ${ }^{4}$. It is used to predict whether the patients have breast cancer or not if yes determines its types. The author first performed CT scan of the images of patients then features are extracted of these CT scan images and final classification was made using ANN. The classification was made in term of the image had cancerous or not. During feed forward the information was given through input layers to output layer and in back propagation the value of the output layers and the output value was compared and result is calculated. The type was determined by the use of BIRADES SHAPE, mass density and mass order. The disease prediction in this case was $90.5 \%$ and health ratio was $80.9 \%$.

Author purposed that early detection of lung cancer can reduce the significant number of breast cancer ${ }^{5}$. The author used Wisconsin breast cancer database, downloaded from UCI Machine repository. 699 original samples were introduced. Two classes were derived from dataset, 458 benign samples and 241 were malignant samples. The hierarchical fuzzy neural network Fuzzy Neural network and fuzzy Gaussian potential neural network 
were used there. The nine features were defined in breast cancer used as an input. Here author concluded that result forms here sensitivity, accuracy and specificity were more accurate than the fuzzy neural networks and also the number of rules were reduced that used in hierarchical neural networks and fuzzy Gaussian potential networks.

In this paper the author used the CT scan images of the lung cancer patients from a private hospital -900 CT scan images of lung cancer images were taken from a private hospital. The author performed experiment was in mat lab. Pre-processing of images was done and then classification of the lung cancer patients in normal and abnormal state was determined. GLCM and BINARIZATION methods were used for features extraction purpose. The supervised feed forward back propagation network was used as a tool for classification purpose and author concluded that after 5 years of lung cancer disease the survival rate of patient was $40 \%$.

In this research paper determined the survival rate of the lung cance ${ }^{7}$. The author improved the survival rate of the lung cancer comparatively previous experiment from $14 \%$ to $49 \%$ of five year of lung cancer patient. First preprocessing of images of lung cancer patients was done for image extraction purpose and 160 features were extracted and Lung field segmentation method was performed on the hidden part of the lung area. Classification was made with the help of neural networks and SVM, for detection and classification of the lung cancer.

Author purposed the environment factors had significant effect on the lung cancer ${ }^{1}$. Author initially took the data of 400 cancer patients from the different diagnostic centres. The pre-processed and clustered of data was done by the used of $\mathrm{k}$ mean algorithm. 400 patients of that which contained 200 lung cancer patients and 200 nonlung cancer patients were taken. There were 200 males and 200 female patients whose age was 20 to 80 .Author developed the lung cancer prediction tool based on data mining that represented the stage of lung cancer patients out of 3 stages and conclude that diagnosed of patient at the last stage mostly impossible so, therefore early prediction of lung cancer should play a vital role in diagnosis of lung cancer.

In this research paper result evaluated that more than one in three people was affected by lung cancer ${ }^{\circ}$. Lung cancer was the leading cause of death related to cancer in many countries. Only $14 \%$ people can survive lung cancer after death. Author used Imagining techniques which combined both positrons emission tomography scan- ner and computer topology scanner in a single system, so the images gathered can be taken as consecutively and combine into a single superpose image. The noise was removed using median filter. The extracted features were stored and different classifications of cancer were determined and those classifications used by physician to give some therapy suggestions.

Here the author focused on detecting cancer on the early stage ${ }^{9}$. First the author removed the Gaussian noise from the CT Scan images of lung cancer patients with the help of non- local mean filter and to segment the lung Otsu's threshing. To form feature vector the textural and structural features were formed from processed images. 3 classifiers SVM, ANN, KNN were used for detection of the lung cancer to found the different stages of the lung cancer and comparison was made between SVM and KNN with respect to accuracy, sensitivity and accuracy. The author concluded that SVM, ANN and KNN had accuracy of $95.12 \%, 92.68 \%$ and $85.37 \%$ respectively for tested images.

The author evaluated among various diseases, the cancer becomes the major thread to India ${ }^{10}$. In India $90 \%$ of the people have cancer who consume tobacco and only $10 \%$ of the people have cancer disease who did not smoke the cancer. Early detection of lung cancer is a key challenge. The author analysed the two techniques neural network and C-mean clustering algorithm. The author concluded that out of these two techniques the neural networks was best to use because the clustering algorithm was not good at low intensity variations.

The author focused on the early stage on the lung cancer. The author took 909 real CT scan of images of lung cancer images ${ }^{\underline{11}}$. Classification was made using neural networks. Classification was made that lung cancer is in normal or abnormal form. After the CT scan of the images, pre-processing of images was done and training and testing were performed along with features extraction. Then classification was done with back propagation neural network and GA algorithm. The author concluded the back propagation neural had the best accuracy to find the early stages of lung cancer after it is passed through GA algorithm. The result was in form of the cancer is the normal or abnormal form.

The lung cancer disease causes a lot of death in world. The cure of the diseases depends upon its initial stages ${ }^{12}$. Lung is usually large in size, so tumours can grows before it can be recognized. So early stages of lung cancer stage 1 and stage 2 are difficult to find. The main objective was 
to found detection of lung cancer before it can grows in a significant level. Images were pre-processed and features have been extracted and final classification of the cancer was made by providing the images as the input to the system with neural networks. The classification was made to identifying the different stages of the lung cancer.

In this paper the author focused on the clustering algorithm that can help to identify the different stages of lung cancer and improvement in medical care ${ }^{13}$. K-mean and farthest first clustering algorithms were used by the author and analysed their performance on the different dataset values. The implementation was done with WEKA software and author concluded that k-mean algorithm was more efficient for lung cancer dataset with arff format.

In this paper the author identified the normal and abnormal stage of lung cancer by used of CT scan of the images ${ }^{14}$. The author used watershed segmentation and thresholding for images segmentation process and watershed technique showed more efficient than the other techniques. The author concluded that lesion size of lung cancer cell is $20 \mathrm{~mm}$ for normal lung cancer and more that $20 \mathrm{~mm}$ as abnormal lung cancer.

\section{Use of Artificial Neural Networks in other Areas}

Artificial Intelligence is playing major role in research of management science and operational research area. It provides ability the machines to behave analytically using some concept. Artificial intelligence has major role in agriculture area, business area, problem solving technique, image processing, accounting data database, computer games and MRI brain tumour analysis ${ }^{15}$.

The author discusses the applications of Artificial Neural Networks in image recognition and classification of crops and weeds 116 The back propagation ANN model was used to differentiate between corn plants from weeds. 80 images including 40 images each of corn plants and weeds were used to provide for training purpose. Kodak DC50 camera was acquired for digitals images of corn plants and weed from McDonald Campus Farm of McGill University. The images were collected from 600 $\mathrm{mm}$ height to capture a bird's s eye view of objects on the ground. Success rate for recognising corn plants was 80 to $100 \%$ where the success rate for recognising weeds was 60 to $80 \%$.
Development of low cost temperature and soil moisture sensor which was placed on the suitable places in the fields and if the soil temperature went above and soil moisture fell below a described limit the water was sprinkled on to the fields ${ }^{17}$. LM-35DZ sensor was used as a water sensor and Probes that was made up of two metal rods tied together using an insulating tape was act as a moisture sensor. The sensor modelling to compensate non-linearity and temperature the Artificial Neural Network to line arise voltage output was used. The ANN consist one input layer, one hidden layer and one output layer. Hidden layer consisted of 4 neurons and output layer consists of 2 nodes. The error rate was concluded less than $1 \%$ that indicated high accuracy of this system.

The author evaluated that three layers Back propagation neural network along with LM algorithm applied to construct a mathematical model that is used in the laser cutting parameters ${ }^{18}$. A genetic algorithm was used to determine the best combination of laser cutting parameters for optimized a specified cutting quality.

In this research paper the author explained the advantages of Artificial Intelligence in the business area ${ }^{19}$. Artificial intelligence system used to make automatic decision which is helpful for companies to using their limited resources to gain more benefits. Artificial Intelligent System helps companies to identify threats and opportunities and make defensive strategies. Artificial Intelligence has significant role in expert system, natural language processing, case based reasoning, decision making and also dangerous tasks for human like mining, fire fighting, bomb disarming can be done with special Artificial Systems.

Artificial intelligence can be used in robots path planning for their independent movement from starting point to target point without any obstacles ${ }^{20}$. For this purpose the ACO (Aunt Colony Optimization) algorithm was used that is based on the phenomenon that aunts follow shortest paths moving from one point to another point. Mitigating stagnation approach was used to overcome the limitations of the Ant Colony Optimization Algorithm which include evaporation, aging and pheromone smoothing approaches. Using ACO algorithm the money can be saved and reliability of the system can be increased.

Author purposed that Artificial Intelligence used for selection of dismantling stations in a vehicle recycling system $^{21}$. The simulation technique using genetic algorithm was used for locating the objects in the networks within 
the limited area. A special expert system was designed for this purpose.

\section{Conclusion}

Artificial neural networks have application in many areas as medical, business, agriculture, for prediction purpose, classification purpose and many others areas. By using artificial neural networks is that the results are more accurate, measurable and errorless. Numbers of challenges are there which involves training to ANN, implementation, interpreting the neural networks and collecting of relevant data. Artificial Neural Networks can use cure and prevent from lung cancer disease and also useful in other industrial area. Performance of ANN can be improved by using suitable optimization technique as Ant colony Optimization.

\section{Acknowledgement}

This paper has been written with the help and guidance of my department. Special thanks to my supervisor Ms. Loveneet Kaur Mangat for their guidelines and experience while writing this review-paper.

\section{References}

1. Ahmed K, Jesmin T, Fatima R, Rahman Z, Ahmed F. Early detection of lung cancer risk using data mining. Asian Pacific Journal of Cancer Prevention. 2012; 14(1):595-8.

2. Biglarian A, Hajizadeh E, Kazemnejada A, Zali MR. Application of artificial neural network in predicting the survival rate of gastric cancer patients. Iran J Public Health. 2011 Apr; 40(2):80-6.

3. Feng $\mathrm{F}, \mathrm{Wu} \mathrm{Y}, \mathrm{Wu} \mathrm{Y}, \mathrm{Nie} \mathrm{G}, \mathrm{Ni}$ R. The effect of artificial neural network model combined with six tumor markers in auxiliary diagnosis of lung cancer. J Med Syst. 2012 sep; 36(5):2973-80.

4. Saritas I. Prediction of breast cancer using artificial neural networks. Journal of Medical Systems. 2012; 36(5):2901-7.

5. Naghibi S, Teshnehlab M. Breast cancer classification based on advanced multi dimensional fuzzy neural network. Journal of Medical Systems. 2012; 36(5):2713-20.

6. Kaur R. Early detection and prediction of lung cancer survival using neural network classifier. IJAIEM. 2013 Jun; 2(6):375-83.

7. Ada, Kaur R. A study of detection of lung cancer using data mining classification techniques. IJARCSSE. 2013 Mar; 3(3):131-4.
8. Nivetha P, Manickavasagam R. Lung cancer detection at early stage using PET/CT imaging technique. International Journal ofAdvance Research in Computer Science and Management Studies. 2014 May; 2(3):3358-63.

9. Naresh R, Shettar R. Early detection of lung cancer using neural network techniques. International Journal of Engineering Research and Management. 2014 Aug; 4(8):78-83.

10. Kaur S. Comparative study review on lung cancer detection using neural network and clustering algorithm. Journal ofAdvance Research in electronicd and Communication. 2014 Feb; 4(2):169-74.

11. Kaur P. Identify lung cancer in its early stage using neural network and ga algorithm. Archives of Physical Medicine and Rehabilitation. 2015 Apr; 4(2):341-4.

12. Hussain A, Ansari T, Gawas P, Chowdhury N. Lung cancer detection using artificial neural network and fuzzy clustering. American Journal of Biomedical Engineering. 2015; 4(3):360-3.

13. Logesh S, Swathy M, Sathish S, Sivaraman J, Rajasekar $M$. Identification of lung cancer cell using watershed segmentation on CT images. Indian Journal of Science and Techonology. 2016 Jan; 9:1-4.

14. Dharmarajan, Velmurugan T. Lung cancer data analysis by $\mathrm{K}$-means and farthest first clustering algorithms. Indian Journal of Science and Techonology. 2105 Jul; 8:1-8.

15. Pannu A. Artificial intelligence and its application in different areas. International Journal of Engineering and Innovative Techonology. 2015 Apr; 4(10):79-84.

16. Yang C, Prasher S, Landry J, Ramaswamy H, Ditommaso A. Application of artificial neural networks in image recognition and classification of crop and weeds. Canadian Agricultural Engineering. 2000; 42(3):147-52.

17. Tyagi A, Reddy A, Singh J, Chowdhury S. A low cost portable temperature-moisture sensing unit with artificial neural network based signals conditioning for smart irrigation applications. International Journal on Smart Sensing and Intelligent Systems. 2011; 4(1):94-111.

18. Chaudhari P, Patel P, Juneja J. Artificial intelligence apply for prediction of laser cutting process - A review. International Journal of Engineering Research and Applicatons. 2012 Aug; 2(4):1025-8.

19. Krishna T. Expert systems in real world business. International Journal of Advance Research in Computer Science and Management Studies. 2013 Dec; 1(7):21-7.

20. Kambam P, Brungi R. Artificial intelligence in robot path planning. IOSR-Journal of Computer Sciences. 2015; 17(3):115-9.

21. Golebiewski B, Trajer J, Choromanski W. System supporting location of service works in agriculture on example of vehicle recycling network. 7th International Scientific Symposium on Farm Machinery and Processes Management in Sustainable Agriculture; 2015. p. 87-93. 